Wesleyan University

From the SelectedWorks of Charles A. Sanislow, Ph.D.

December, 2005

\title{
Preliminary evidence for medication effects on functional abnormalities in the amygdala and anterior cingulate in bipolar disorder
}

Hilary P. Blumberg

Nelson H. Donegan

Charles A. Sanislow

Susan Collins

Cheryl Lacadie, et al. 
Hilary P. Blumberg • Nelson H. Donegan - Charles A. Sanislow • Susan Collins • Cheryl Lacadie - Pawel Skudlarski • Ralitza Gueorguieva • Robert K. Fulbright • Thomas H. McGlashan · John C. Gore · John H. Krystal

\section{Preliminary evidence for medication effects on functional abnormalities in the amygdala and anterior cingulate in bipolar disorder}

Received: 3 April 2005 / Accepted: 14 July 2005 / Published online: 26 October 2005

(C) Springer-Verlag 2005

\begin{abstract}
Rationale: Abnormal amygdala and frontocortical responses to emotional stimuli are implicated in bipolar disorder (BD) and have been proposed as potential treatment targets. Objectives: The aim of this study was to investigate amygdala and frontocortical responses to emotional face stimuli in $\mathrm{BD}$ and the influences of mood-stabilizing medications on these responses. Methods: Functional magnetic resonance imaging was performed while $17 \mathrm{BD}$ participants (5 unmedicated) and 17 healthy comparison (HC) participants viewed faces with happy, sad, fearful, or neutral expressions. Results: The group by stimulus-condition interaction was significant $(p<0.01)$ for amygdala activation, with the greatest effects in the happy face condition. Relative to $\mathrm{HC}$, amygdala increases were greater in unmedicated $\mathrm{BD}$, but lower in medicated BD. Rostral anterior cingulate (rAC) activation was decreased in unmedicated $\mathrm{BD}$ compared to $\mathrm{HC}$;
\end{abstract}

H. P. Blumberg $(\bowtie) \cdot$ N. H. Donegan · C. A. Sanislow ·

S. Collins · T. H. McGlashan - J. H. Krystal

Mood Disorders Research Program, Department of Psychiatry,

Yale University School of Medicine,

300 George Street, Suite 901,

New Haven, CT 06511, USA

e-mail: hilary.blumberg@yale.edu

Tel.: +1-203-7856180

Fax: $+1-203-7372513$

H. P. Blumberg · C. Lacadie · P. Skudlarski · R. K. Fulbright Department of Diagnostic Radiology,

Yale University School of Medicine,

New Haven, CT 06519, USA

H. P. Blumberg · J. H. Krystal

Depression Research Center (REAP),

Department of Veterans Affairs, VA CT Healthcare System,

West Haven, CT 06516, USA

R. Gueorguieva

Department of Epidemiology and Public Health,

Yale University,

New Haven, CT 06520, USA

J. C. Gore

Department of Radiology, Vanderbilt University,

Nashville, TN 37232, USA however, BD participants taking medication demonstrated rAC activation similar to $\mathrm{HC}$ participants. Conclusions: Although the sample sizes were small, these preliminary results suggest that $\mathrm{BD}$ is associated with increased amygdala and decreased $\mathrm{rAC}$ response to emotional faces. The findings also provide preliminary evidence that mood-stabilizing medications may reverse abnormalities in BD in the response of an amygdalafrontal neural system to emotional stimuli.

Keywords Bipolar disorder - Functional magnetic resonance imaging $\cdot$ Amygdala $\cdot$ Frontal lobe $\cdot$ Lithium carbonate $\cdot$ Anticonvulsant

\section{Introduction}

Amygdala and ventral frontal cortex are major nodes in the neural circuitry subserving emotional responses. These structures share reciprocal connections that permit them to work together to modulate adaptive responses to shifts in emotional stimuli. Preclinical studies, as well as human studies of patients with medial temporal lobe seizures and lesions in ventral frontal cortex, implicate elevations in amygdala activity and deficits in frontocortical activity in the impaired emotional regulation characteristic of bipolar disorder (BD) (Blumberg et al. 2002).

Neuroimaging studies provide further support for the presence of functional abnormalities in an amygdalafrontal neural system in BD. Elevations in resting activity of the amygdala in BD depression (Drevets et al. 2002), as well as elevations in amygdala response to negative and positive emotional faces in BD patients in mixed, depressed and euthymic states, have been reported (YurgelunTodd et al. 2000; Lawrence et al. 2004). Disturbances in ventral frontal resting activity and recruitment during performance of emotional and executive tasks have been demonstrated in BD individuals in manic, depressed, as well as euthymic mood states (Blumberg et al. 1999, 2003; Drevets et al. 1997; Rubinsztein et al. 2001; Lawrence et al. 2004; Strakowski et al. 2004). Emerging evidence suggests 
that normalization of these functional disturbances in frontotemporal neural systems by anticonvulsants and lithium may contribute to their mood-stabilizing properties (Drevets et al. 2002; Goodwin et al. 1997; Ketter and Wang 2002; Lawrence et al. 2004). This study employed an emotional face paradigm to probe amygdala and ventral frontal responses to emotional stimuli in $\mathrm{BD}$ and to explore effects of medications on regional brain responses.

\section{Methods}

\section{Participants}

Participants included 17 outpatients with BD I treated at the Veterans Affairs Connecticut Healthcare System or the Yale University School of Medicine or by practitioners in the community. The participants with BD I (see Table 1 for demographic and clinical features of the participants with BD) were subdivided into a group that was unmedicated at the time of scanning [UNMED BD, $N=5,3(60 \%)$ female, ages $23-56$ years, mean age $40.0 \pm \mathrm{SD} 12.3 ; 1(20 \%)$ in a mixed state, $2(40 \%)$ depressed, and $2(40 \%)$ euthymic at scanning; 2 (40\%) met criteria for rapid cycling] and a group that was medicated at the time of scanning (MED BD, $N=12$, 4 (33\%) female, ages 29-59, mean 45.8 \pm SD9.4; 4 $(33 \%)$ in manic, hypomanic, or mixed states, $1(8 \%)$ depressed, 7 (58\%) euthymic; 5 (42\%) rapid cycling. The healthy comparison (HC) group consisted of 17 participants who were without history of Axis I disorders themselves or in first-degree relatives [HC, 10 (59\%) female, ages 20-55, mean 33.2 \pm 10.8$]$. Structured clinical interviews confirmed the presence or absence of Diagnostic and Statistical Manual of Mental Disorders, Fourth Edition Axis I diagnoses (First et al. 1995). All participants were right-handed with the exception of two BD participants. The Hamilton Depression Rating Scale (HDRS) (Williams et al. 1994) and the Clinician-Administered Rating Scale for Mania (CARSM) (Altman et al. 1994) quantified mood symptoms at the time of scanning. Psychotropic medications used by the MED BD participants included anticonvulsants $(N=8,67 \%)$ [valproic acid $(N=6,50 \%)$, gabapentin $(N=2,17 \%)$, carbamazepine $(N=1,8 \%)]$, lithium $(N=4,33 \%)$, antidepressants $(N=3,25 \%)$, and atypical antipsychotics $(N=1,8 \%)$. BD participants were without comorbidity with the exception of treated hypothyroidism (one MED BD participant taking levothyroxine) and distant (remission $>1.5$ years) history of alcohol dependence [two (40\%) UNMED BD, nine (75\%) MED BD participants]. Written informed consent was obtained from all participants in accordance with the human investigation committees of the Yale University School of Medicine and Department of Veterans Affairs.

Functional magnetic resonance imaging procedures

Imaging methods and activation paradigm were as described previously (Donegan et al. 2003). In brief, 16 coronal-oblique echoplanar images were acquired $(\mathrm{TR}=1,650$ $\mathrm{msec}, \mathrm{TE}=60 \mathrm{msec}$, flip angle $=60^{\circ}, \mathrm{FOV}=20 \times 20 \mathrm{~cm}, 64 \times 64$ matrix, $3.1 \times 3.1 \mathrm{~mm}$ in-plane resolution $\times 7 \mathrm{~mm}$ slice thickness) from the frontal pole through the fusiform region with the 8th slice centered on the amygdala while participants passively viewed stimuli presented at a rate of $1 / \mathrm{s}$. Twentysecond blocks of happy, sad, fearful, or neutral faces from the Ekman face series (Ekman and Friesen 1979), or a cross-hair fixation point (baseline block), were presented in an order counterbalanced to control for sequential dependencies during eight $4 \mathrm{~min} 20 \mathrm{sec}$ runs. Images were corrected for motion, spatially filtered (Gaussian filter $6.25 \mathrm{~mm}$ full-width half maximum), and the first two images of each block discarded to account for hemodynamic delay. The percent signal change for each of the four emotional face conditions, as compared to the baseline

Table 1 Demographic and clinical features of the participants with bipolar disorder

\begin{tabular}{llllll}
\hline Sex & Age (years) & Handedness & Medication & Mood state & Rapid-cycling \\
\hline Female & 23 & Right & None & Mixed & Yes \\
Female & 44 & Right & None & Euthymic & Yes \\
Female & 56 & Right & None & Euthymic & No \\
Male & 34 & Right & None & Depressed & No \\
Male & 43 & Right & None & Depressed & No \\
Female & 45 & Right & Lithium & Mixed & Yes \\
Female & 55 & Right & Lithium & Euthymic & Yes \\
Male & 35 & Right & Lithium, levothyroxine & Euthymic & No \\
Male & 59 & Right & Lithium, sertraline & Depressed & Yes \\
Female & 38 & Left & Valproic acid & Euthymic & Yuthymic \\
Female & 40 & Right & Valproic acid & Manic & Yes \\
Male & 29 & Right & Valproic acid & Euthymic & No \\
Male & 42 & Left & Gabapentin, carbamazepine & Euthymic & No \\
Male & 48 & Right & Valproic acid, quetiapine & No \\
Male & 48 & Right & Valproic acid, sertraline & Euthymic & No \\
Male & 53 & Right & Gabapentin, venlafaxine & Hypomanic & No \\
Male & 58 & Right & Valproic acid & Manic & \\
\hline
\end{tabular}


condition, were averaged across runs for each participant. Signal change maps for each individual were coregistered to T1-weighted anatomical images obtained immediately prior to the echoplanar images and transformed into standardized stereotactic space (Talairach and Tournoux 1988). The amygdala region of interest (ROI) and a control region of fusiform gyrus anticipated to activate in response to all faces were predefined within Talairach space (Donegan et al. 2003), and a mean percent signal change was calculated separately for the right and left amygdala and fusiform ROIs for each participant. Ventral frontal differences were hypothesized; however, we did not constrain a region in ventral prefrontal cortex a priori and performed between-group comparison maps of signal change by calculation of $t$ statistics at all pixels. A rostral anterior cingulate (rAC) ROI was identified as the only region that differed significantly ( $p<0.005$, cluster threshold of 20 adjacent voxels) between BD participants and HC subjects. This rAC ROI was used in analyses to assess for effects of demographic and clinical factors.

\section{Analyses}

Statistical procedures were performed in SAS version 8.1 (SAS Institute Inc., Cary, NC) using a mixed-model analysis (PROC MIXED). In the primary analysis, mean percent signal change in the amygdala ROI for each subject was the dependent measure. Group was the between-subjects variable (three levels: UNMED BD, MED BD, HC). The emotional face condition (happy, sad, fearful, neutral) and hemisphere (right, left) were the within-subject variables, and there was a random subject effect and condition within subject effect. Repeated measures were performed for hemisphere. Terms that were not significant were eliminated via a backward stepwise algorithm, with the constraint that the model at each step was hierarchically well formulated. Least square means (ls means) and standard errors (SE) were calculated in the mixed model and plotted to interpret significant interactions. Analogous analyses were performed for the rAC ROI, as well as for the fusiform comparison ROI, as the dependent measures. Results were considered significant at the level of $p<0.025$, providing Bonferroni correction for the two ROIs investigated.

Posthoc analyses were conducted to explore potential main effects of clinical variables on amygdala and rAC measures by adding relevant factors to the model one at a time including age, sex, mood state at scanning, rapid cycling, history of alcohol dependence, and HDRS or CARS-M mood rating scale scores, as well as age of onset, duration of illness and number of hospitalizations. To explore the effects of medication class, the MED BD group was further divided into subjects who were prescribed anticonvulsants and subjects who were prescribed lithium, and the models were refit with a four-category group variable rather than a three-category group variable. Posthoc adjusted pairwise comparisons were performed to explain significant interactions or main effects.
Significant results from these analyses are reported below.

\section{Results}

Age

There was a significant main effect of age $[F(2,31)=5.13$, $p=0.01]$, derived from significant difference between the HC and MED BD groups $(p=0.003)$. Age did not differ significantly between other groups $(p>0.2)$.

\section{Amygdala}

The group by stimulus-condition interaction was significant after Bonferroni correction $[F(6,93)=2.84, p=0.01]$, with significant group differences detected in the happy condition. In this condition, the amygdala activation of the UNMED BD group was higher than that of the HC group, whereas the amygdala activation of the MED BD group was lower than that of the HC group (UNMED BD ls mean percent signal increase \pm SE $0.18 \pm 0.06 ;$ HC $0.04 \pm 0.03$; MED BD $-0.07 \pm 0.04$ ) (Figs. 1 and 2). Effect of group was not significant $(p>0.4)$ within the other emotional conditions.

\section{Rostral anterior cingulate}

The ROI analysis confirmed the group comparison map analysis (group effect, $[F(2,31)=9.61, p<0.001]$ ). The highest activation was in the HC group (HC $0.04 \pm 0.04$ ), the lowest values in the unmedicated BD group (UNMED $\mathrm{BD}-0.28 \pm 0.07)$, and intermediate values were detected in the medicated BD group (MED BD $-0.04 \pm 0.04$ ) (Figs. 3 and 4). There was a trend toward a greater effect of group in the left hemisphere $[F(2,133)=3.30, p=0.04$, uncorrected $]$.

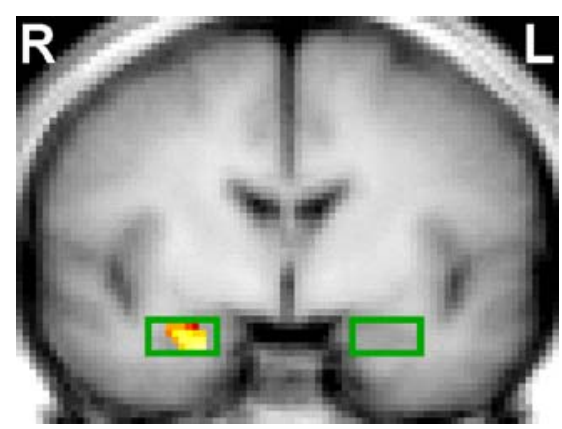

Fig. 1 Increased amygdala activation during happy face viewing in unmedicated BD. Coronal image (plane $4 \mathrm{~mm}$ posterior to the anterior commissure) demonstrates increased amygdala activation (green box demonstrates the a priori amygdala region of interest) in the unmedicated BD group as compared to the healthy control group, significance level $p<0.05$. The maximum of this difference was at coordinates $x=25 \mathrm{~mm}, y=-4 \mathrm{~mm}, z=-15 \mathrm{~mm}$ (Talairach and Tournoux 1988). $R$ Right brain, $L$ left brain 


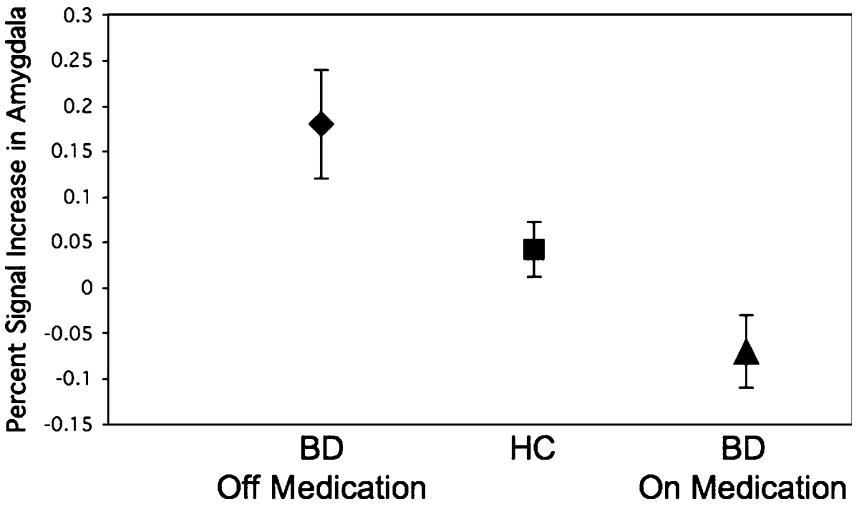

Fig. 2 Blunting of elevated amygdala activation during happy face viewing in $\mathrm{BD}$ in association with medication. The graph displays the group ls mean percent signal increase and SE values for amygdala activation during the happy face condition in the unmedicated BD group $(N=5), \mathrm{HC}$ group $(N=17)$, and medicated BD group $(N=12)$

Fusiform gyrus

The fusiform region activated across groups in response to face stimuli; the effect of group was not significant $[F(2,31)=0.12, p=0.89]$.

\section{Medication class}

Exploration for effects of medication classes revealed a significant association, after Bonferroni correction, between the BD subgroup prescribed anticonvulsants and reduced amygdala activity in the happy condition $[F(1,81.7)=13.88, p=0.0008]$ (amygdala activation in BD taking anticonvulsants $-0.10 \pm 0.05$ ), as compared to UNMED BD. Amygdala activation was also lower in the BD subgroup prescribed lithium than the UNMED BD group; however, this effect did not reach significance $[F(1,81.7)=4.40, p=0.078]$. In rAC, higher $\mathrm{rAC}$ activation was associated with the BD subgroup prescribed lithium

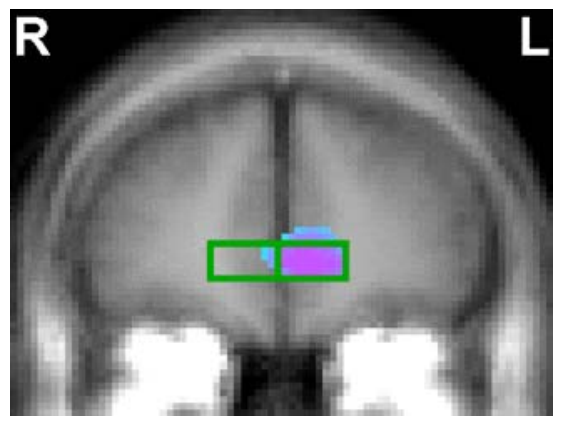

Fig. 3 Decreased rAC activation during face viewing in unmedicated BD. Coronal image (plane $40 \mathrm{~mm}$ anterior to the anterior commissure) demonstrates the relative decrease in $\mathrm{rAC}$ activation (green box demonstrates the rAC region of interest used for the post hoc analyses) in the unmedicated BD group as compared to the $\mathrm{HC}$ group, significance level $p<0.005$. The maximum of this difference was at coordinates $x=-6 \mathrm{~mm}, y=40 \mathrm{~mm}, z=7 \mathrm{~mm}$ (Talairach and Tournoux 1988). $R$ Right brain, $L$ left brain

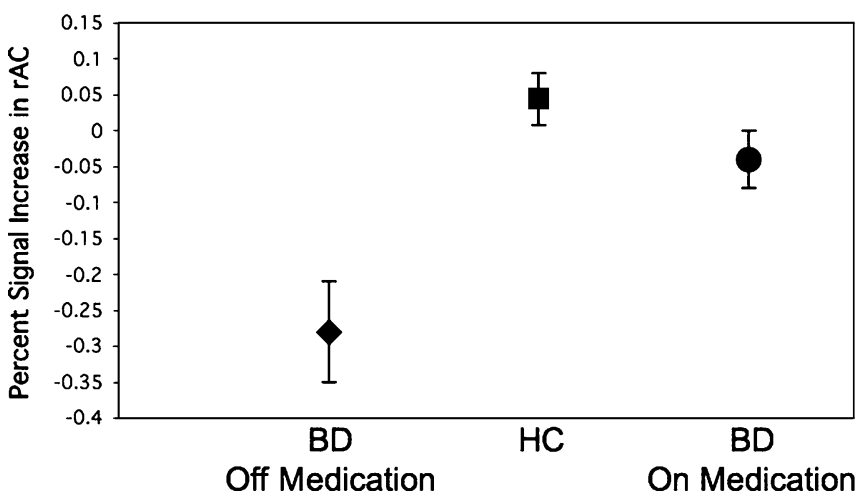

Fig. 4 Attenuation of $\mathrm{rAC}$ activation decreases during face viewing in $\mathrm{BD}$ in association with medication. The graph displays the group ls mean percent signal increase and SE values for rAC activation during face viewing in the unmedicated BD group $(N=5)$, HC group $(N=17)$, and medicated BD group $(N=12)$

$[F(1,30)=-3.62, p=0.006]$ (rAC activation in $\mathrm{BD}$ prescribed lithium $0.06 \pm 0.07$ ) as compared to UNMED BD.

\section{Other clinical factors}

No significant effects were detected for age, sex, mood state, rapid cycling, history of alcohol dependence, HDRS or CARS-M scores, age of onset, duration of illness, or number of hospitalizations.

\section{Discussion}

We found differences between the frontotemporal responses to emotional face stimuli in unmedicated $\mathrm{BD}$ compared to $\mathrm{HC}$ participants. These differences were attenuated in the presence of pharmacotherapy (medicated BD subgroup). Amygdala activation was elevated in unmedicated BD participants, especially when they viewed positive face stimuli, but was blunted to levels below those of $\mathrm{HC}$ in medicated BD participants, particularly if they were taking anticonvulsants. In contrast, activation in rAC in unmedicated BD participants was lower than that in $\mathrm{HC}$ participants. This difference was attenuated in medicated BD participants, especially if they were taking lithium, as they had higher rAC activation than unmedicated BD participants and activation similar to $\mathrm{HC}$ participants.

The finding of elevated amygdala response to emotional face stimuli in $\mathrm{BD}$ is consistent with previous reports (Yurgelun-Todd et al. 2000; Lawrence et al. 2004). Whereas in unipolar depression, elevated amygdala activation has been observed in response to faces depicting negative affect; in BD, elevated amygdala activation has been observed in response to faces depicting positive as well as negative affect (Lawrence et al. 2004). The strong effect found in this study in association with the viewing of happy faces is consistent with theories that response to positively valenced emotional stimuli may be especially salient in BD (Leibenluft et al. 2003). Lawrence et al. (2004) have suggested that BD may be characterized by an en- 
hanced perception of salience of both positive and negative emotional stimuli that may contribute to mood lability in the disorder. We did not detect elevations in amygdala response in these BD participants to faces depicting negative emotional expressions, as have been noted in previous studies of BD participants (Yurgelun-Todd et al. 2000, Lawrence et al. 2004). Possible explanations include limitations in the power to detect differences owing to the small sample size, as well as the inclusion of BD participants with elevated mood symptoms, as attenuated amygdala response to faces depicting negative affect have been reported in association with manic symptoms in BD (Lennox et al. 2004).

The lower amygdala response observed in BD participants taking mood-stabilizing medications is consistent with reductions in amygdala hyperactivity observed previously in depressed BD participants treated with anticonvulsants (Drevets et al. 2002). These findings are consistent with theories that attenuation of abnormally elevated amygdala activity, e.g., through the reduction of excitatory glutamatergic input and facilitation of gamma aminobutyric acid transmission, contributes to the therapeutic effects of moodstabilizing medications (Post et al. 1982; Drevets et al. 2002; Krystal et al. 2002). It is notable that amygdala response to positive face stimuli in patients taking moodstabilizing medication was lower than that in HC. Potential blunting of response to positive stimuli could be an important area for future study as BD patients often report the blunting of their perceptions of positive stimuli while taking mood-stabilizing medications as the reason for discontinuing their medication (Goodwin and Jamison 1990).

The $\mathrm{rAC}$ region, where attenuated response to emotional faces was observed in unmedicated BD participants, shares substantial reciprocal connections with the amygdala and, together with amygdala, subserves emotional processes (Amaral and Price 1984; Devinsky et al. 1995). An inverse relationship between frontal deficits and elevated amygdala response in mood disorders has been reported previously (Davidson 2000; Siegle et al. 2002). It is possible that deficits in rAC could contribute to disinhibition of the amygdala or that abnormal activity in the amygdala could lead to decreases in rAC response. Elucidation of mechanisms underlying this imbalance may provide useful insights into the pathophysiology of mood disorders.

Activation of the rAC in medicated BD participants was similar to that in HC. Metabolic changes in rAC in association with lithium response have been reported (Davanzo et al. 2001). Normalization of prefrontal response to emotional stimuli in BD with lithium was also observed previously in a small sample of patients $(N=3)$ with $\mathrm{BD}$ (Lawrence et al. 2004). It is unclear whether frontal changes in association with medication contribute to symptom reduction. In one study, such changes were not associated with recovery from acute mood symptoms (Goodwin et al. 1997), and conclusions regarding beneficial treatment responses in $\mathrm{BD}$ cannot be drawn from the current study design. However, the rAC region identified in this study overlaps the region demonstrated by multiple groups studying major depressive disorder (with much larger samples and more systematic study) to be associated with treatment response (e.g., Mayberg 2002). Thus, future studies with larger BD samples and more systematic investigation of medications and clinical outcomes are warranted.

The ability to draw conclusions from this patient sample of modest size is limited. Mood-congruent biases in BD (Murphy et al. 1999; Lembke and Ketter 2002) could have contributed to the findings. Heterogeneity within the participant sample and the naturalistic treatment design limit the causal inferences that can be made about the associations between pharmacotherapy and regional brain abnormalities in BD. Differences detected between BD subgroups could reflect preexisting differences between BD participants that could have influenced their adherence to different types of medication or clinical factors that may have influenced the choice of pharmacotherapy by treating physicians. Future studies with BD participants stratified by mood state and studied prospectively with randomized medication assignment are warranted.

In summary, this study provides evidence to support the presence of abnormalities in the response of an amygdalarAC neural system to emotional face stimuli in BD. The data suggest the importance of considering the valence of emotional stimuli in studies of BD. They also suggest that mood-stabilizing medications used to treat BD may normalize functional abnormalities within frontotemporal neural systems.

Acknowledgements The authors thank Kathleen Colonese, BA, for her expert care in coordinating the research; Hedy Sarofin, RTRMR, and Terry Hickey, RTRMRN, for their technical expertise; and the research subjects for their participation.

The authors were supported by research grants from the National Alliance for Research in Affective Disorders and Schizophrenia (Great Neck, NY) (HPB), The Ethel F. Donaghue Women's Investigator Program at Yale (New Haven, CT) (HPB), the Borderline Personality Disorder Research Foundation (NHD, CAS, THM), the Department of Veterans Affairs Research Career Development (HPB), Merit Review (HPB) and Research Enhancement Award Program (REAP) (HPB, JHK) Awards, Alcohol Research Center (JHK) and Clinical Neurosciences Division of the National Center for PTSD (JHK) (Washington, DC) and the National Institute of Mental Health R01MH69747 and R01MH070902 (HPB), National Institute on Alcohol Abuse and Alcoholism K05 AA 14906-01 (JHK) and NIH/NIMH K05-01654 (THM) (Bethesda, MD).

\section{References}

Altman EG, Hedeker DR, Janicak PG, Peterson JL, Davis JM (1994) The clinician-administered rating scale for mania (CARS-M): development, reliability, and validity. Biol Psychiatry $36: 124-134$

Amaral DG, Price JL (1984) Amygdalo-cortical projections in the Monkey (Macaca fascicularis). J Comp Neurol 230:465-496

Blumberg HP, Stern E, Ricketts S, Martinez D, de Asis J, White T, Epstein J, Isenberg N, McBride PA, Kemperman I, Emmerich S, Dhawan V, Eidelberg D, Kocsis JH, Silbersweig DA (1999) Rostral and orbital prefrontal cortex dysfunction in the manic state of bipolar disorder. Am J Psychiatry 156:1986-1988

Blumberg HP, Charney DS, Krystal JH (2002) Frontotemporal neural systems in bipolar disorder. Semin Clin Neuropsychiatry $7: 243-254$ 
Blumberg HP, Leung HC, Skudlarski P, Lacadie C, Fredericks C, Harris B, Charney DS, Gore JC, Krystal JH, Peterson BS (2003) A functional magnetic resonance imaging study of bipolar disorder: state- and trait-related dysfunction in ventral prefrontal cortices. Arch Gen Psychiatry 60:601-609

Davanzo P, Thomas MA, Yue K, Oshiro T, Belin T, Strober M, McCracken J (2001) Decreased anterior cingulate myo-inositol/ creatine spectroscopy resonance with lithium treatment in children with bipolar disorder. Neuropsychopharmacology 24: 359-369

Davidson RJ (2000) Affective style, psychopathology, and resilience: brain mechanisms and plasticity. Am Psychol 55:11961214

Devinsky O, Morrell MJ, Vogt BA (1995) Contributions of anterior cingulate cortex to behaviour. Brain 118:279-306

Donegan NH, Sanislow CA, Blumberg HP, Fulbright RK, Lacadie C, Skudlarski P, Gore JC, Olson IR, McGlashan TH, Wexler BE (2003) Amygdala hyperreactivity in borderline personality disorder: implications for hyper vigilance and emotional dysregulation. Biol Psychiatry 54:1284-1293

Drevets WC, Price JL, Simpson JR, Todd RD, Reich T, Vannier M, Raichle ME (1997) Subgenual prefrontal cortex abnormalities in mood disorders. Nature 386:824-827

Drevets WC, Price JL, Bardgett ME, Reich T, Todd RD, Raichle ME (2002) Glucose metabolism in the amygdala in depression: relationship to diagnostic subtype and plasma cortisol levels. Pharmacol Biochem Behav 71:431-447

Ekman P, Friesen WV (1979) Pictures of facial affect. Consulting Psychologists, Palo Alto, CA

First MB, Spitzer RL, Gibbon M, Williams JBW (1995) Structured clinical interview for DSM-IV Axis I \& II disorders (Version 2.0). New York State Psychiatric Institute, New York

Goodwin FK, Jamison KR (1990) Manic-depressive illness. Oxford University Press, New York

Goodwin GM, Cavanagh JT, Glabus MF, Kehoe RF, O'Carroll RE, Ebmeier KP (1997) Uptake of 99mTc-exametazime shown by single photon emission computed tomography before and after lithium withdrawal in bipolar patients: associations with mania. Br J Psychiatry 170:426-430

Ketter TA, Wang PW (2002) Predictors of treatment response in bipolar disorders: evidence from clinical and brain imaging studies. J Clin Psychiatry 63(Suppl 3):21-25

Krystal JH, Sanacora G, Blumberg H, Anand A, Charney DS, Marek G, Epperson CN, Goddard A, Mason GF (2002) Glutamate and GABA systems as targets for novel antidepressant and mood-stabilizing treatments. Mol Psychiatry 7(Suppl 1):S71-S80
Lawrence NS, Williams AM, Surguladze S, Giampietro V, Brammer MJ, Andrew C, Frangou S, Ecker C, Phillips ML (2004) Subcortical and ventral prefrontal cortical neural responses to facial expressions distinguish patients with bipolar disorder and major depression. Biol Psychiatry 55:578-587

Leibenluft E, Charney DS, Pine DS (2003) Researching the pathophysiology of pediatric bipolar disorder. Biol Psychiatry 53:1009-1020

Lembke A, Ketter TA (2002) Impaired recognition of facial emotion in mania. Am J Psychiatry 159:302-304

Lennox BR, Jacob R, Calder AJ, Lupson V, Bullmore ET (2004) Behavioural and neurocognitive responses to sad facial affect are attenuated in patients with mania. Psychol Med 34:795-802

Mayberg HS (2002) Modulating limbic-cortical circuits in depression: targets of antidepressant treatments. Semin Clin Neuropsychiatry 7:255-268

Murphy FC, Sahakian BJ, Rubinsztein JS, Michael A, Rogers RD, Robbins TW, Paykel ES (1999) Emotional bias and inhibitory control processes in mania and depression. Psychol Med 29:1307-1321

Post RM, Uhde TW, Putnam FW, Ballenger JC, Berrettini WH (1982) Kindling and carbamazepine in affective illness. J Nerv Ment Dis 170:717-731

Rubinsztein JS, Fletcher PC, Rogers RD, Ho LW, Aigbirhio FI, Paykel ES, Robbins TW, Sahakian BJ (2001) Decision-making in mania: a PET study. Brain 124:2550-2563

Siegle GJ, Steinhauer SR, Thase ME, Stenger VA, Carter CS (2002) Can't shake that feeling: event-related fMRI assessment of sustained amygdala activity in response to emotional information in depressed individuals. Biol Psychiatry 51:693-707

Strakowski SM, Adler CM, Holland SK, Mills N, DelBello MP (2004) A preliminary fMRI study of sustained attention in euthymic, unmedicated bipolar disorder. Neuropsychopharmacology 29:1734-1740

Talairach J, Tournoux P (1988) Co-planar stereotaxic atlas of the human brain. Thieme Medical Publishers, New York

Williams JBW, Link MJ, Rosenthal NE, Amira L, Terman M (1994) Structured interview guide for the Hamilton depression rating scale: seasonal affective disorder version (SIGH-SAD). New York State Psychiatric Institute, New York

Yurgelun-Todd DA, Gruber SA, Kanayama G, Killgore WD, Baird AA, Young AD (2000) fMRI during affect discrimination in bipolar affective disorder. Bipolar Disord 2:237-248 